ARTICLE

\title{
Correlative 3D microscopy of single cells using super-resolution and scanning ion-conductance microscopy
}

Vytautas Navikas ${ }^{1,7}$, Samuel M. Leitao 2,7, Kristin S. Grussmayer (10 1,6, Adrien Descloux (D) 1, Barney Drake ${ }^{2}$ Klaus Yserentant (1) ${ }^{3}$, Philipp Werther ${ }^{4}$, Dirk-Peter Herten (1) ${ }^{3}$, Richard Wombacher (10 4,5, Aleksandra Radenovic (i) ${ }^{1 凶} \&$ Georg E. Fantner (i) ${ }^{2 凶}$

High-resolution live-cell imaging is necessary to study complex biological phenomena. Modern fluorescence microscopy methods are increasingly combined with complementary, label-free techniques to put the fluorescence information into the cellular context. The most common high-resolution imaging approaches used in combination with fluorescence imaging are electron microscopy and atomic-force microscopy (AFM), originally developed for solidstate material characterization. AFM routinely resolves atomic steps, however on soft biological samples, the forces between the tip and the sample deform the fragile membrane, thereby distorting the otherwise high axial resolution of the technique. Here we present scanning ion-conductance microscopy (SICM) as an alternative approach for topographical imaging of soft biological samples, preserving high axial resolution on cells. SICM is complemented with live-cell compatible super-resolution optical fluctuation imaging (SOFI). To demonstrate the capabilities of our method we show correlative 3D cellular maps with SOFI implementation in both 2D and 3D with self-blinking dyes for two-color high-order SOFI imaging. Finally, we employ correlative SICM/SOFI microscopy for visualizing actin dynamics in live COS-7 cells with subdiffraction-resolution.

\footnotetext{
${ }^{1}$ Laboratory of Nanoscale Biology, Institute of Bioengineering, School of Engineering, Swiss Federal InstSlitute of Technology Lausanne (EPFL), Lausanne, Switzerland. ${ }^{2}$ Laboratory for Bio- and Nano-Instrumentation, Institute of Bioengineering, School of Engineering, Swiss Federal Institute of Technology Lausanne (EPFL), Lausanne, Switzerland. ${ }^{3}$ College of Medical and Dental Sciences, Medical School \& School of Chemistry, University of Birmingham, Birmingham, United Kingdom. ${ }^{4}$ Institute of Pharmacy and Molecular Biotechnology, Heidelberg University, Heidelberg, Germany. ${ }^{5}$ Department of Chemical Biology, Max Planck Institute for Medical Research, Heidelberg, Germany. 'Present address: Grussmayer Lab, Department of Bionanoscience, Faculty of Applied Science and Kavli Institute for Nanoscience Delft, Delft University of Technology, Delft, Netherlands. ${ }^{7}$ These authors contributed equally: Vytautas Navikas, Samuel M. Leitao. ${ }^{凶}$ email: aleksandra.radenovic@epfl.ch; georg.fantner@epfl.ch
} 
maging living cells in vitro is crucial in deciphering the biochemical mechanisms underlying complex cellular activity such as cell motility ${ }^{1}$, differentiation ${ }^{2}$, membrane trafficking ${ }^{3}$, and cell-to-cell communication ${ }^{4}$. Our knowledge about the ultrastructure of a cell is almost exclusively derived from highresolution electron microscopy (EM) on fixed and sectioned cells. Modern EM techniques can routinely reach sub-nm resolution and even provide the atomic structures of macromolecular complexes such as ribosomes ${ }^{5}$ or lately even whole viruses ${ }^{6}$. The function of the structures is typically inferred from the presence of specific molecules of interest by performing targeted expression or immunolabelling, followed by fluorescence microscopy techniques thus resulting in correlative light and electron microscopy $(\text { CLEM })^{7}$. Axial information can be obtained from EM images through serial sectioning ${ }^{8}$, however obtaining unperturbed $3 \mathrm{D}$ information about the shape of the cell membrane remains difficult and low throughput.

The portfolio of fluorescence imaging techniques has been expanded with multiple so-called super-resolution microscopy techniques, that circumvent the traditional diffraction limit of optical microscopy ${ }^{9}$. Many of these techniques trade-off temporal resolution for lateral resolution, and often require high light intensities causing light-induced cell damage in live cells ${ }^{10}$. An approach named super-resolution optical fluctuation imaging $(\mathrm{SOFI})^{11}$ was developed to mitigate the negative phototoxic effects of long-term imaging by increasing acquisition speeds. SOFI relies on stochastic temporal fluctuations of the signal in independently blinking emitters and is less sensitive to varying fluorophore density and different blinking conditions, compared to localization microscopy approaches ${ }^{12}$. SOFI was demonstrated as a powerful imaging method which goes well beyond the diffraction limit and can be extended for live-cell 3D imaging ${ }^{13}$ or combined with self-blinking dyes ${ }^{14}$, simplifying the image acquisition pipeline.

On the other hand, scanning probe microscopy (SPM) can obtain nanometer resolution images of the $3 \mathrm{D}$ surface of unlabeled living cells in their physiological environment ${ }^{15}$. It has been proven as a versatile tool for biological imaging, and is often combined with fluorescence microscopy methods ${ }^{16}$. The most commonly used SPM technique, atomic-force microscopy (AFM), enables to study of the mechanobiology of fixed or living cells ${ }^{17}$, to image biological membranes ${ }^{18}$ and even track the dynamics of molecular assembly processes ${ }^{19}$. Label-free AFM methods combined with label-specific super-resolution fluorescence microscopy tools can provide maps of single-cells in unprecedented detail not only in fixed, but also in living samples ${ }^{20,21}$. AFM relies on the direct physical interaction with a sample, which usually deforms sensitive biological specimens and causes height artifacts in live-cell imaging 22 . To perform truly non-contact imaging in physiological conditions a scanning modality based on ionic current sensing was developed a few decades $\mathrm{ago}^{23}$ and was significantly improved with further developments for robust live-cell imaging ${ }^{24}$. Scanning-ion conductance microscopy (SICM) relies on the ionic current flowing through a nanocapillary in electrolyte. The ionic current strongly depends on the presence of a surface in the vicinity of the probe tip, hence the nanocapillary can be used as a nanoscale proximity sensor, without ever touching the sample. The sensing distance where the current drops is dependent on the pipette's pore diameter ${ }^{25}$. SICM is therefore suitable for sensitive biological systems such as neurons, even with high-aspect ratio topographies with the invention of the hopping mode scanning modality ${ }^{24}$.

The use of combined SICM and stimulated emission depletion microscopy for topographical imaging was demonstrated to provide additional information about the structure of cytoskeletal components $^{26}$, but lacked the ability to perform the complementary correlative recording on the same setup. Here we present a combined SICM-SOFI approach for in-situ, correlative single-cell imaging. We first demonstrate our combined SICMSOFI approach on dedicated setups for $2 \mathrm{D}$ and $3 \mathrm{D}$ high-order SOFI microscopy. We then use it to study subcellular morphology by using correlative information with subdiffraction resolution obtained sequentially. Finally, the established SICM-SOFI simultaneous measurement pipeline allow us to visualize actin dynamics in live cells in a correlative manner.

\section{Results}

Correlative SICM and SOFI imaging pipeline. Our pipeline for a combined SICM/SOFI approach is based on correlative imaging with a home-built high speed SICM setup (Fig. 1a), operating in hopping mode ${ }^{24}$, combined with a SOFI workflow for either twoor three-dimensional sample imaging. SOFI increases the resolution of the widefield microscope by using the statistical information from stochastic intensity fluctuations and calculating the cumulants of the intensities between the individual pixels and image planes for resolution improvement in $2 \mathrm{D}$ and $3 \mathrm{D}$ respectively ${ }^{11}$, (Fig. 1b). The lateral resolution of SICM is approximately equal to three times the radius of the pipette ${ }^{25}$. In this work, we exclusively use nanocapillaries with a radius of 40-60 nm, which results in a lateral resolution comparable to SOFI. For increased resolution, glass nanocapillaries smaller than $10 \mathrm{~nm}$ radius can be fabricated ${ }^{27}$. Such nanocapillaries are however highly susceptible to clogging and are therefore not suitable for long-term measurements.

To get label-specific information about the cytoskeletal structure of the cells, we performed SOFI with both traditional and self-blinking fluorescent dyes. Self-blinking dyes enable highorder SOFI analysis, that can be applied to various densities of temporally fluctuating fluorophores in order to gain spatial resolution and improve optical sectioning ${ }^{14}$. SOFI has the advantage of tolerating higher labeling densities ${ }^{12}$ compared to single-molecule localization microscopy, thus decreasing the acquisition time required to the order of $10 \mathrm{~s}$ of seconds per SOFI frame ${ }^{28}$. Subsequently, we correlated SICM data with twocolor $2 \mathrm{D}$ and $3 \mathrm{D}$ SOFI images, that provided high-resolution single-cell maps showing a distribution of the actin and tubulin cytoskeletal proteins (Fig. 1c).

2D SOFI and SICM correlative imaging. Before combining the two techniques within one instrument, we tested the 2D SOFI approach with self-blinking dyes on a dedicated setup (Fig. 2a, b). We achieved up to $72 \pm 3 \mathrm{~nm}$ resolution for $4^{\text {th }}$ order SOFI images for microtubules labeled with the commercially available Abberior FLIP-565 dye and $101 \pm 9 \mathrm{~nm}$ for actin labeled with custom synthesized $\mathrm{f}-\mathrm{HM}-\mathrm{SiR}^{29}$, compared to a widefield resolution of $437 \pm 106 \mathrm{~nm}$ and $480 \pm 30 \mathrm{~nm}$ (mean \pm s.d., $\mathrm{N}=8$ images for SOFI 2D resolution measurements) respectively. Resolution was estimated using an image decorrelation analysis algorithm ${ }^{30}$, which allowed us to estimate the resolution imagewise. The calculated values agreed with theoretical values expected from SOFI analysis (Supplementary Fig. 2). Low-intensity $\left(275 \mathrm{~W} / \mathrm{cm}^{2}\right.$ of $632 \mathrm{~nm}, 680 \mathrm{~W} / \mathrm{cm}^{2}$ for $\left.561 \mathrm{~nm}\right)$ illumination was used, resulting in minimal bleaching and long bleaching lifetimes of $406 \pm 168 \mathrm{~s}$ for Abberior FLIP-565 and $625 \pm 130 \mathrm{~s}$ for f-HMSiR (mean \pm s.d, $N=8$ images for SOFI $2 \mathrm{D}$ bleaching measurements) (Supplementary Fig. 3). Labeling density for both dyes was optimized for SOFI imaging, however both datasets were also processed with SMLM software ${ }^{31}$, which revealed that SOFI analysis was comparable in terms of resolution to the SMLM approach in low SNR conditions. Furthermore, analysis showed 

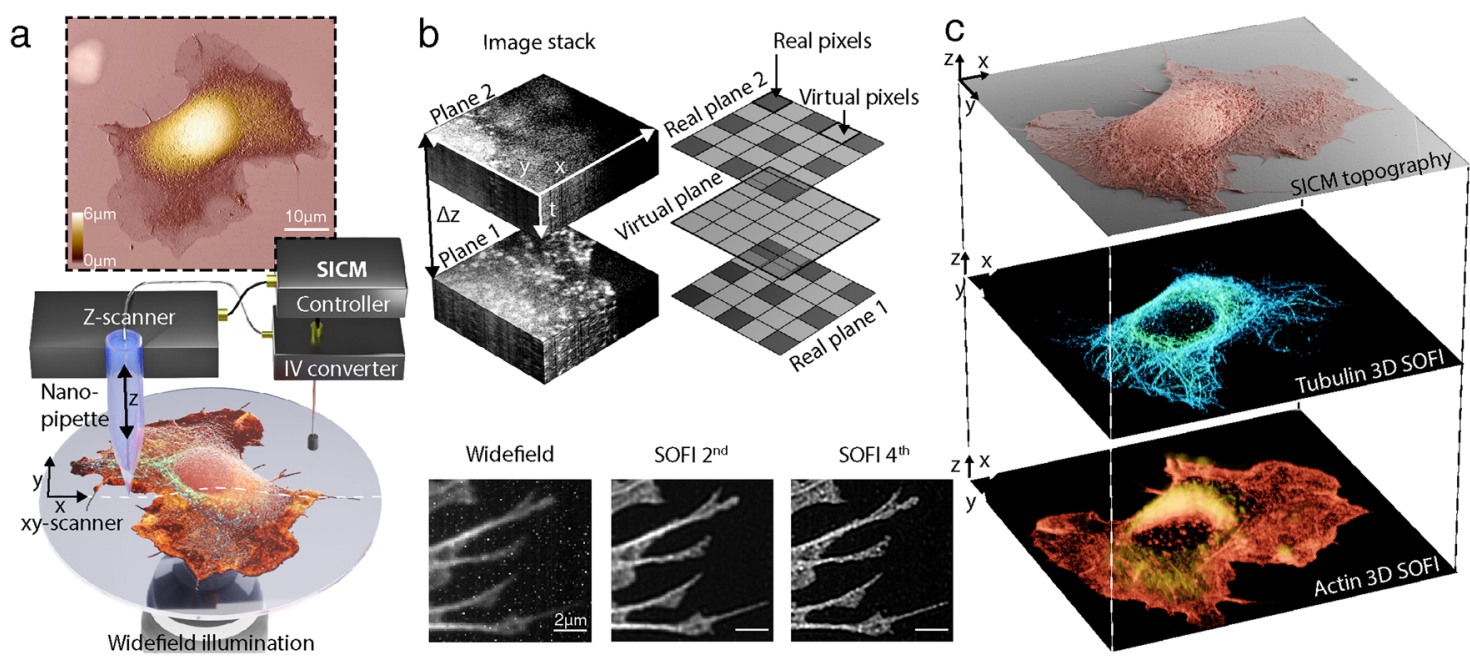

Fig. 1 Combined scanning ion conductance microscopy (SICM) and super-resolution optical fluctuation imaging (SOFI) for three-dimensional topography-fluorescence correlative imaging. a Schematic illustration of the SICM-SOFI setup. In SICM, a current is generated through a nanopipette and modulated as a function of the z-position. The image generated reflects a three-dimensional topographical view of the surface at high lateral and axial resolution (top panel). The lateral resolution in SICM ranges from $150 \mathrm{~nm}$ to $30 \mathrm{~nm}$ and the axial resolution is in the $10 \mathrm{~nm}$ range (Supplementary Fig. 1). Since the scanning head is mounted on top of the widefield setup, fluorescence imaging can be performed simultaneously. $\mathbf{b}$ Conceptual visualization of the SOFI principle. The recorded image stack, containing time traces of independently fluctuating fluorophores is used for analysis. A SOFI image is generated by computing cross-cumulants, creating virtual pixels between adjacent real pixels. The cross-cumulant computation principle can be applied in the axial direction as well if multiple sample planes are simultaneously acquired. For the $n$th order SOFI image, the cumulant point-spread function volume is raised to $n$th power thus giving the resolution improvement of a factor of $\sqrt{n}$. The inset images of the actin structure at the bottom of the (b) panel represent a standard deviation of the image stack (widefield), $2^{\text {nd }}$ and $4^{\text {th }}$ order of linearized SOFI images with corresponding lateral resolution values estimated with an image decorrelation-analysis algorithm of $420 \mathrm{~nm}, 194 \mathrm{~nm}$, and $93 \mathrm{~nm}$, respectively. c Schematics of correlative SICM and two-color 3D SOFI imaging.

artefacts in high-density regions, non-optimal for SMLM analysis. The resolution, estimated with an image decorrelation analysis algorithm, was found to be $56 \mathrm{~nm}$ for tubulin and $98 \mathrm{~nm}$ for actin (Supplementary Fig. 4). The excellent optical sectioning and contrast provided by SOFI allowed to routinely resolve individual actin filaments (Fig. 2a, Supplementary Fig. 5). Subsequently, the corresponding cells were imaged in a custom SICM using an adaptive hopping mode ${ }^{32}$. This yielded the topographical map of the cell membrane (Fig. 2c) resolving individual microvilli and filopodia membrane structures. Fluorescence and SICM images were registered based on the features from the topography map and the SOFI actin channel (Fig. 2d). Filamentous actin (f-actin) is known to be located in the lower part of the cell volume and correlates well with the topography of the cell boundary ${ }^{33}$ in COS-7 cells, that allowed us to simplify the registration process (Supplementary Fig. 6).

Multiplane 3D SOFI and SICM correlative imaging. SOFI is not limited in providing the resolution improvement laterally, but it can also be used to improve axial resolution and sectioning by using information from multiple image planes acquired simultaneously. To demonstrate 3D SOFI's capability of volumetric single cell imaging, we performed two-color 3D SOFI imaging on a dedicated setup for the same cytoskeleton components with a multiplane SOFI approach based on an image-splitting prism $^{34}$ (Fig. 3a, b), allowing us to calculate cumulants in 3D. However, the trade-off for $3 \mathrm{D}$ imaging capability is a reduced signal-tonoise ratio, which makes it difficult to perform high-order SOFI analysis, therefore we only show up to $3^{\text {rd }}$ order 3D SOFI images of microtubules labeled with the Abberior FLIP-565 dye and actin labeled with Alexa-647 (Supplementary Fig. 7). The acquired 8 physical planes resulted in 22 total planes spaced $116 \mathrm{~nm}$ apart after $3^{\text {rd }}$ order SOFI computation, giving a 3D volume of $50 \mu \mathrm{m} \times$ $60 \mu \mathrm{m} \times 2.45 \mu \mathrm{m}$ with a lateral resolution of $151 \pm 34 \mathrm{~nm}$ for tubulin structures and $168 \pm 34 \mathrm{~nm}$ for Alexa 647 labeled f-actin which was comparable to comparable to the $3^{\text {rd }}$ order $2 \mathrm{D}$ SOFI results (Supplementary Fig. 2). A single-color 3D SOFI image required as little as $3 \mathrm{~min}$ to acquire. This facilitates the screening of a large number of 3D cell volumes (Supplementary Fig. 8).

We then correlated the $3 \mathrm{D}$ SOFI information with the $3 \mathrm{D}$ SICM images, which have an order of magnitude higher axial resolution. The samples were moved to the dedicated SICM setup (Fig. 4a) to resolve microvilli at the surface of the cell (Fig. 4b, c). Finally, we correlated SICM and 3D SOFI information (Fig. 4d), which resulted in a detailed picture of the cell volume showing actin colocalized with microvilli protrusions over the whole cell surface (Fig. 4e).

Correlative information for subcellular morphology. We have further interpreted the correlative information and compared the topographical maps of multiple cellular structures with two fluorescent channels which we acquired. Taking advantage of a multiplane SOFI approach, we compared the SICM topography with volumetric localization of actin (Fig. 5a) and tubulin (Fig. 5b) in a vertical section, which is marked in Figs. $3 c$ and $4 a$. Patches of actin are distributed in the top and bottom parts of the cell, while tubulin is distributed homogenously within the cell volume. We also measured the correlation values for different cellular structures such as filopodia (Fig. 5c), microvilli (Fig. 5d) and microtubules (Fig. 5e) and calculated Pearson correlation coefficients between the pairs of actin, tubulin and SICM topography channels. The physical access to microtubules by SICM was obtained by partially removing the top membrane using Triton X-100 detergent during the permeabilization step $^{35}$ (Supplementary Fig. 9). We found high correlations between the SICM/actin channels for filopodia and microvilli structures and for SICM/ tubulin channels (Fig. 5f) for the chemically unroofed cell.

Combined 2D SOFI and SICM for live-cell imaging. The establishment of a correlative SOFI/SICM pipeline on different 

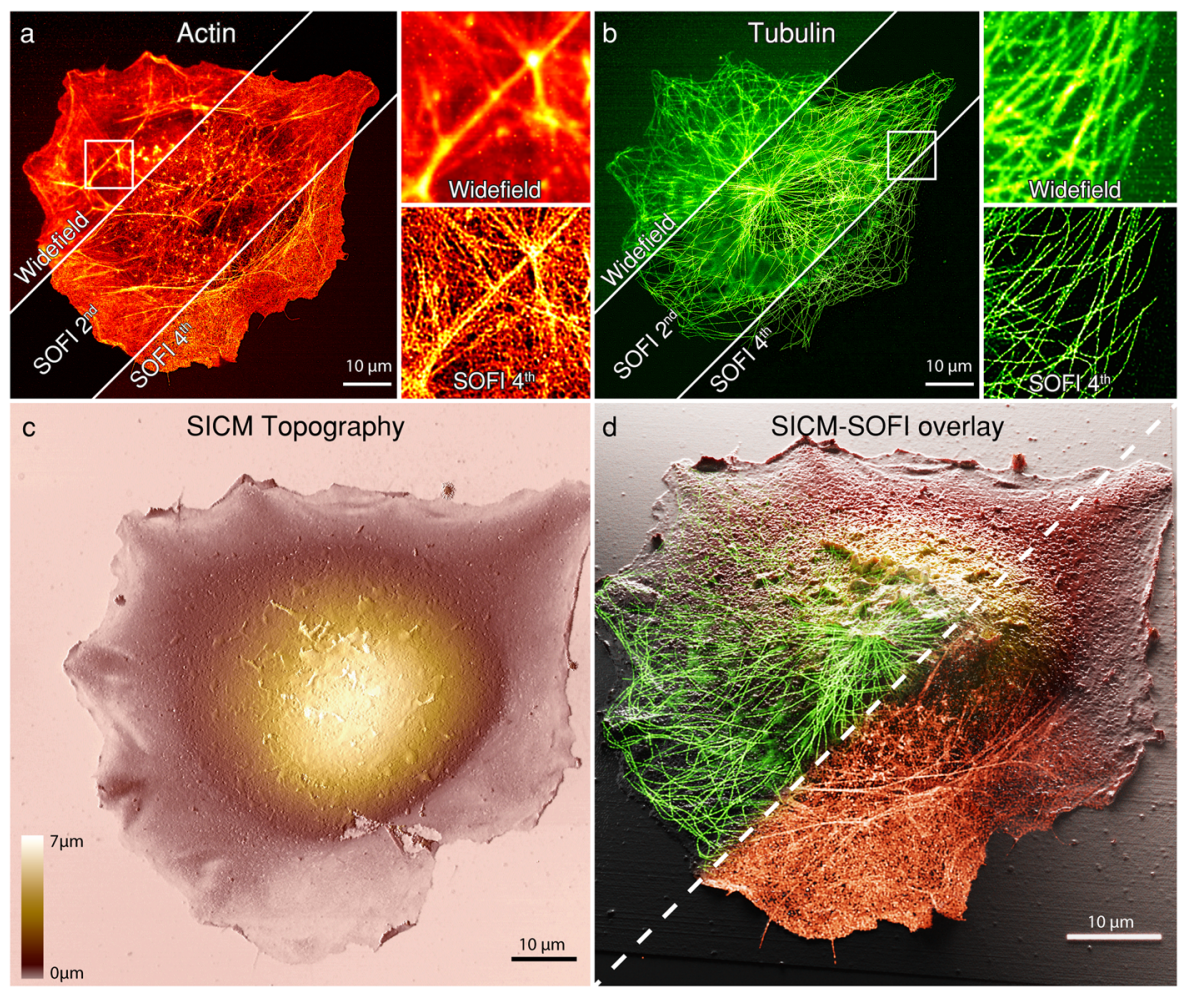

Fig. 2 Correlative 2D SICM-SOFI imaging cytoskeletal components of a fixed COS-7 cell. a Large field of view $(100 \times 100 \mu \mathrm{m}) 2 \mathrm{D}$ SOFI imaging of filamentous actin labeled with a phalloidin-f-HM-SiR self-blinking dye conjugate. b Subsequently, immunostained tubulin labeled with the self-blinking Abberior FLIP-565 dye was imaged. $10 \mu \mathrm{m} \times 10 \mu \mathrm{m}$ zoom-ins of the standard deviation of the image stacks (widefield) and $4^{\text {th }}$ order SOFI images are shown for $\mathbf{a}$ and $\mathbf{b}$ panels. $635 \mathrm{~nm}$ flat-fielded laser excitation was used for imaging f-HM-SiR and a $561 \mathrm{~nm}$ laser line for Abberior FLIP-565. Imaging was performed in a $25 \%$ glycerol and PBS mixture at $\mathrm{pH}=8$. c Corresponding topographical SICM image of the same cell acquired on a dedicated SICM setup. $1024 \times 1024$ pixels image ( $78 \mathrm{~nm}$ pixel size), acquired at a pixel acquisition rate of $200 \mathrm{~Hz}$ with a hopping height of $5 \mu \mathrm{m}$. $\mathbf{d} 3 \mathrm{D}$ rendering of a correlative SICM-SOFI overlay. The transparency gradients of tubulin and actin channels are shown as a 2D plane, while topographical SICM information is used for the height representation in the Blender 3D software. The overlay shows that actin is distributed within the boundary of the cell while tubulin displays the canonical cytoskeletal structure. This figure is representative of more than 10 correlative 2D SICM/SOFI single-cell two-color images obtained from at least two independent experiments.
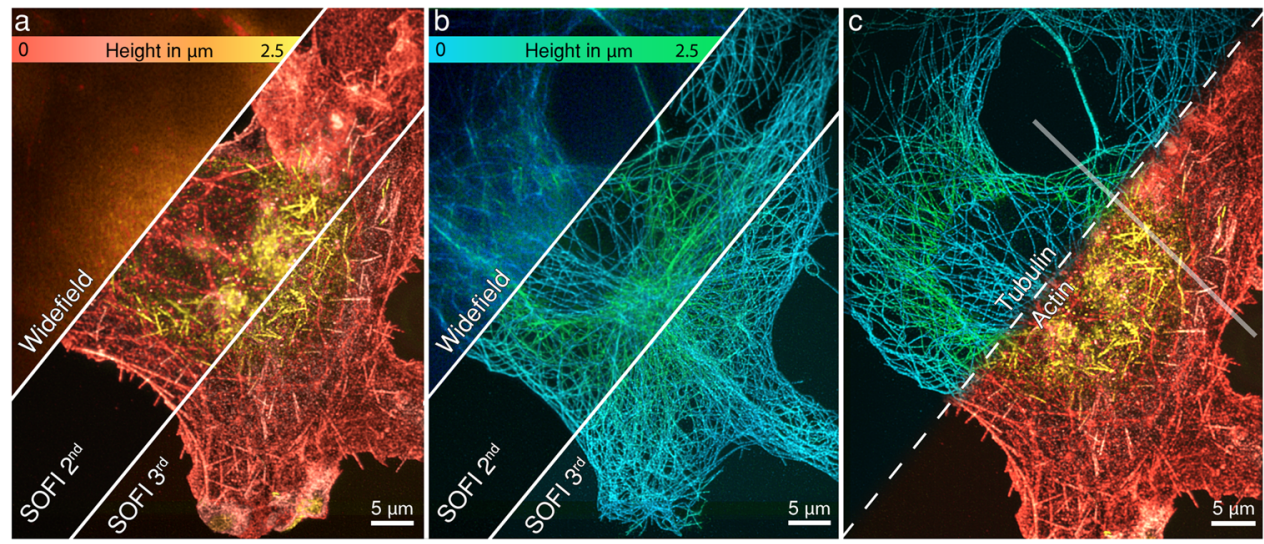

Fig. 3 Multi-plane 3D SOFI imaging of cytoskeletal components in a fixed COS-7 cell. a 3D SOFI imaging of filamentous actin labeled with phalloidin conjugated to Alexa-647 and tubulin (b) labeled with the Abberior FLIP-565 self-blinking dye. A sample volume of $2.45 \mu \mathrm{m} \times 65 \mu \mathrm{m} \times 55 \mu \mathrm{m}$ was recorded with 8 equally spaced physical planes, resulting in 22 image planes after $3^{\text {rd }}$ order SOFI processing. Flat-fielded $635 \mathrm{~nm}$ and $532 \mathrm{~nm}$ laser lines were used for the excitation. Alexa-647 dye was imaged in reductive/oxidative system imaging buffer with $10 \mathrm{mM}$ mercaptoethylamine, while the Abberior FLIP-565 dye was imaged in a 50\% glycerol and PBS solution at $\mathrm{pH}=7.5$. Height is represented in color scales displayed for each channel. c Co-registered $3 \mathrm{D} 3^{\mathrm{rd}}$ order SOFI volumes of tubulin and filamentous actin. Co-registration was performed based on brightfield microscopy images acquired after recording each fluorescence channel. Semi-transparent line shows the cross-sections displayed in Fig. 5 (a, b). This figure is representative of more than 10 two-color 3D SOFI images obtained from at least two independent experiments. 

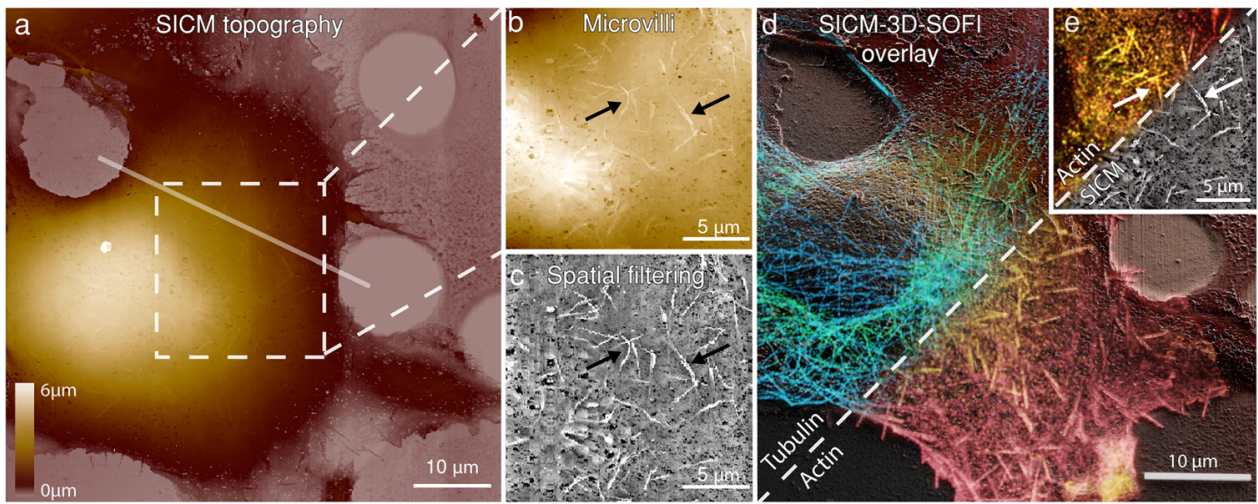

Fig. 4 Correlative SICM and 3D SOFI imaging of cytoskeletal components in a fixed COS-7 cell. a Topographical SICM map of a fixed COS-7 cell imaged on a dedicated SICM setup after two-color 3D-SOFI acquisition. Corresponding fluorescence images are shown in Fig. 3. The scan resolution is $1024 \times 1024$ pixels over an $80 \mu \mathrm{m} \times 80 \mu \mathrm{m}$ area with a corresponding pixel size of $78 \mathrm{~nm}$. The pixel acquisition rate was $200 \mathrm{~Hz}$ with a hopping height of $6 \mu \mathrm{m}$. Semitransparent line shows the cross-section area displayed in Fig. $5(\mathbf{a}, \mathbf{b}) . \mathbf{b}$ Leveled zoom-in of the upper part of the cell by mean plane subtraction, showing the topography of microvilli (marked with black arrows) on the surface of the cell. c Spatial band-passed filtered SICM image to highlight multiple microvilli structures. d 3D rendered correlative SICM and two-color 3D-SOFI overlay. The tubulin and actin channels are rendered as volumes consisting of 22 planes, while topographical SICM information is used for a height representation in the Blender 3D software (Supplementary Movie 1). e Correlative SOFI and SICM overlay (white arrows correspond to the black arrows in $\mathbf{b}$ and $\mathbf{c}$ ). This figure is representative of more than 10 correlative SICM and 2-color 3DSOFI single-cell images obtained from at least two independent experiments.

instruments paved the way for a combined instrument. For correlative live-cell imaging, we combined the prototype SICM instrument with a 2D SOFI capable widefield microscope. We performed a correlative measurement of cytoskeletal proteins and cell morphology revealing the correlated dynamics of actin and membrane topography at the subcellular level. We transfected COS-7 cells for cytoskeletal proteins of actin filaments (actinin or actin) fused with mEOS-2 photo-switching fluorescent proteins in order to achieve stochastic fluctuations in the fluorescence signal. Cells were then scanned in a consecutive manner with SICM and imaged with SOFI in a custom-design chamber with an environmental control ${ }^{32}$ (Supplementary Fig. 10). Recordings of 300 frame-long stacks of the fluorescence signal were performed after each SICM frame, resulting in a combined SICM/SOFI acquisition time of $3 \mathrm{~min} /$ frame for images of parts of the cell, and 10 min for whole cell imaging (Supplementary Figs. 11 and 12). The $2^{\text {nd }}$ order SOFI images in Fig. 6a show the actin filaments with a $173 \pm 20 \mathrm{~nm}$ (mean \pm s.d, $N=6$ images for live-cell SOFI $2 \mathrm{D}$ resolution measurements) lateral resolution. After recording, SICM and SOFI images were aligned, revealing correlated dynamics of cytoskeletal proteins and membrane topography (Fig. 6b, c). During the SICM imaging, there was no laser applied, resulting in two-minute dark intervals between SOFI frames. This significantly reduced the photodamage enabling us to record time-lapse sequences of 10-15 SOFI frames for up to $42 \mathrm{~min}$ without observable cell negative phototoxic effects (Supplementary Fig. 11).

\section{Discussion}

We have established correlative membrane topography and cytoskeleton imaging provided by combined SICM and SOFI modalities for fixed COS-7 cells both in $2 \mathrm{D}$ and $3 \mathrm{D}$ on separate imaging setups preserving the state-of-the-art capabilities of both imaging modalities. For imaging densely-labeled samples we used self-blinking dyes suitable for high-order SOFI imaging. Additionally, the 3D SOFI approach allowed us to retrieve information about the cytoskeletal protein distribution within the cell volume. Subsequently acquired SICM high-resolution axial topography provided detailed volumetric cell-mapping. Finally, we have performed simultaneous SICM/SOFI live-cell imaging in a combined setup for routinely obtaining correlative measurements in vitro.

Previous studies have demonstrated that a combination of topographical and biochemical sample information is a powerful tool which can provide a comprehensive picture of cellular activity. Correlative SPM and super-resolution microscopy studies, involving techniques such as SMLM $^{36}$, stimulated emission depletion (STED) ${ }^{26,37}$, structured light illumination (SIM) ${ }^{21}$ have been demonstrated. SOFI, as a computational method, does not require a complex optical setup design compared to SIM or STED approaches. We show that it can be easily implemented on existing SPM/SICM setups with only few optical components required. Simultaneous SICM measurements can be used to directly detect the cell topography changes related to apoptosis ${ }^{38}$ thus acting as a detector for phototoxicity. Furthermore SICM has been shown to outperform AFM in live-cell imaging ${ }^{22}$ due to its non-contact nature, which is crucial in imaging sensitive samples. SICM and confocal microscopy combination was demonstrated to be a versatile tool to study virus-like particle and cell interactions ${ }^{39,40}$. Small imaging volume, located in the vicinity of the pipette, allows to have superior optical sectioning and colocalized measurement, which is ideal for studying cellular activity near the membrane. On the other hand, AFM has demonstrated its superiority over SICM in terms of imaging resolution ${ }^{41}$. AFM is also more versatile in terms of retrieving information about the nanomechanical sample properties ${ }^{42}$ and can even be used for molecular-specific imaging 43 . Due to the fragile nature of the glass nanocapillary, SICM is less forgiving of imaging mistakes that can lead to fracture of the capillary. This is exacerbated by the fact that no pre-fabricated SICM capillaries are commercially available.

We demonstrated the ability of the combined method for biological studies of the dynamic cell morphology and cytoskeletal architecture inside the cells. This could be used to investigate open questions in membrane trafficking ${ }^{3}$, cell migration ${ }^{44}$ or infection. Due to the electrical nature of SICM measurements, it can also retrieve surface charge information ${ }^{45}$, thus adding an extra dimension for the measurement. The ability to additionally map the charge of a cell membrane is particularly relevant for problems such as clustering of membrane proteins ${ }^{46}$, membrane curvature influence on density of membrane proteins and 

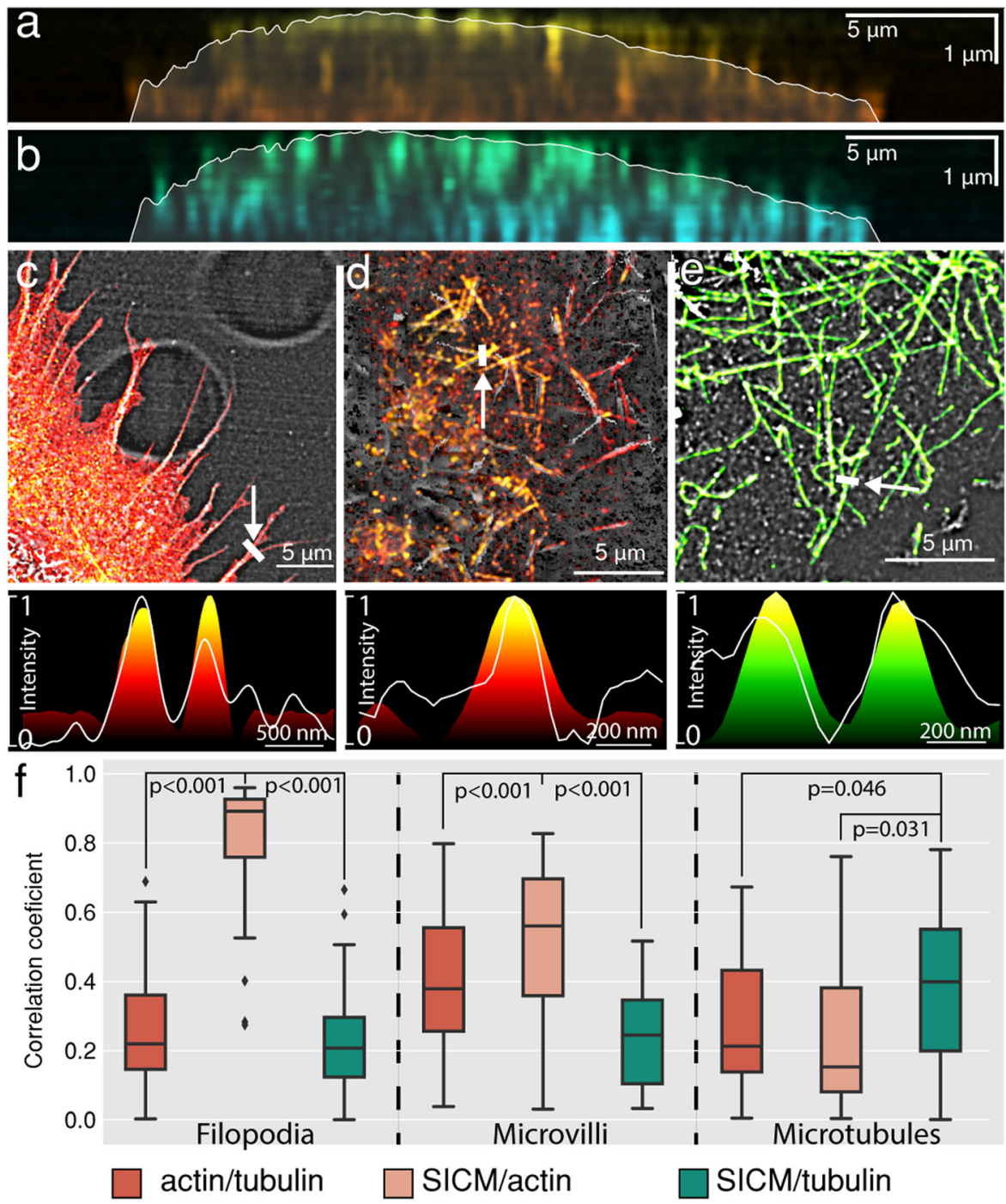

Fig. 5 Volumetric distribution of cytoskeletal components from correlative SICM-3D-SOFI images. Vertical cross-sections of actin (a) and tubulin (b) in a fixed COS-7 cell marked in Figs. 3c and 4a overlaid with the SICM topography displayed as a semi-transparent contour. Correlative imaging of different cellular components resolved by SICM: filopodia overlaid with fluorescently labeled f-actin from 3D-SOFI (c), microvilli overlaid with fluorescently labeled factin from 3D-SOFI (d) and microtubules of chemically unroofed COS-7 cells overlaid with fluorescently labeled tubulin from 3D-SOFI (e). Normalized intensity profiles are displayed below each image. The arrows in c-e are guides for the eye to indicate the locations of cross sections. For overlays only, the intensity values of SOFI images were projected on spatially filtered SICM images for a better structural representation. $\mathbf{f}$ Normalized Pearson crosscorrelation coefficients of normalized height and intensity cross-sections for both SICM topography and fluorescence channels. Cross-sections ( $N=30$ ) were manually selected (Supplementary Fig. 13) from the SICM image for each of the features in 3 different correlative images. Correlation coefficients were measured on filipodia structures, microvilli structures and microtubules. Significantly higher $(p<0.05$, two-sided $t$-test $)$ correlation values were identified for filopodia(SICM)/actin (2D-SOFI), microvilli (SICM)/actin (3D-SOFI) and microtubules (SICM)/tubulin (3D-SOFI). For all comparisons, $p$ values are shown in the upper part of $\mathbf{f}$ panel. The lower and upper bounds of the box plot correspond to the first and third quartiles, while the middle line shows the median. The upper and lower whiskers extend no further than 1.5 times of the interquartile range (IQR) from upper and lower bounds. Points outside this range are defined as outliers. Raw source data are provided in the Source Data File. This figure is representative of more than 10 correlative SICM and 2-color 2D/3D SOFI single-cell images obtained from at least two independent experiments.

lipids $^{47}$, lipid-rafts ${ }^{48}$, behavior of voltage-gated ion channels ${ }^{49}$, etc. While there are some remaining technological challenges in terms of usability, the combination of the multimodal SICM and flexible SOFI has the potential to become a routine live-cell imaging modality capable of tackling challenging biological problems.

\section{Methods}

2D widefield fluorescence imaging setup. A home-built widefield microscopy setup described previously ${ }^{14}$ was used (Supplementary Fig. 14). The setup has four laser lines for illumination: a $200 \mathrm{~mW} 405 \mathrm{~nm}$ laser (MLL-III-405-200mW, Roithner Lasertechnik), a 1 W 632 nm laser (SD-635-HS-1W, Roithner
Lasertechnik), a $350 \mathrm{~mW} 561 \mathrm{~nm}$ laser (Gem561, Laser Quantum) and a $200 \mathrm{~mW}$ $488 \mathrm{~nm}$ laser (iBEAM-SMART-488-S-HP, Toptica Photonics). The beam of the $635 \mathrm{~nm}$ laser is flat fielded by coupling it into the multimode fiber and passed through a speckle reducer (Optotune, LSR-3005-17S-VIS) similarly to that described previously ${ }^{50}$. All laser lines are collimated, expanded and focused in the back focal plane of the water immersion 60x objective (Nikon SR Plan Apo IR 60x $1.27 \mathrm{NA}$ WI) resulting in the effective FOV of $100 \times 100 \mu \mathrm{m}$ with a pixel size of $107 \mathrm{~nm}$. Fluorescence signal is then filtered using a quad-line dichroic mirror (ZET405/488/561/640, AHF Analysetechnik) and emission filter (R405/488/561/ 635 flat, AHF Analysetechnik). Additional band-pass emission filters (596/83 or $685 / 70$, Chroma) were used for sequential two-color imaging. Finally, the light is focused on a sCMOS camera (ORCA Flash 4.0, Hamamatsu; back projected pixel size of $108 \mathrm{~nm})$. Z stabilization is achieved with a PID controller using a total internal reflection from a coverslip using $980 \mathrm{~nm}$ laser diode (CPS980, Thorlabs) 

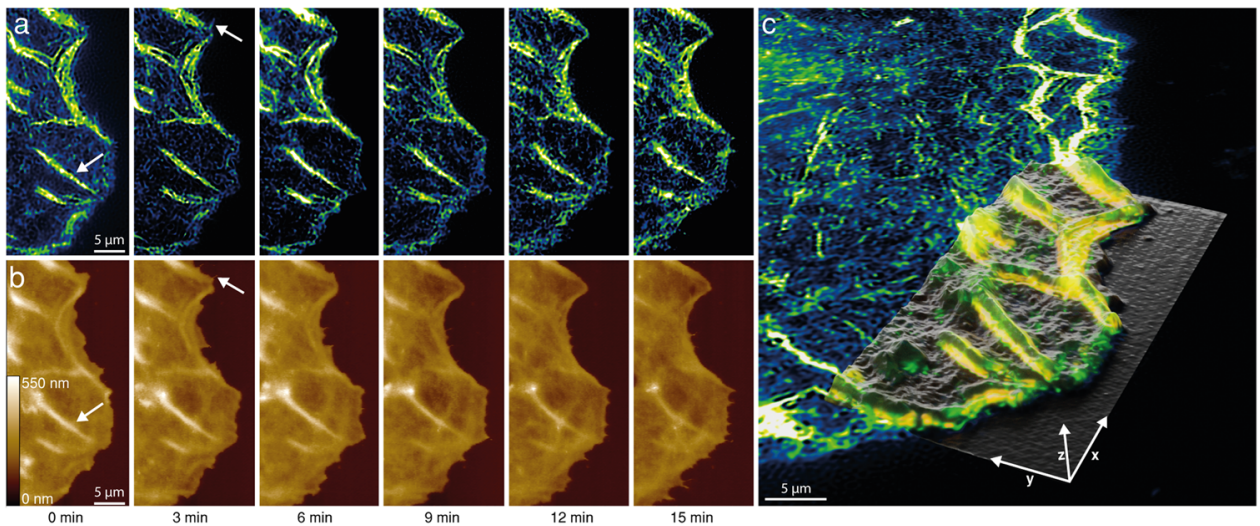

Fig. 6 Live-cell SICM/SOFI imaging of cytoskeletal actinin dynamics of COS-7 cell. a $2^{\text {nd }}$ order SOFI images of actinin-mEOS-2 in transfected COS-7 cells reconstructed from 250 frames each, thus allowing to achieve $12.5 \mathrm{~s}$ temporal resolution per SOFI frame. b Corresponding SICM height maps (200 $\times 100$ pixels image, with $200 \mathrm{~nm}$ pixel size) were acquired at a pixel acquisition rate of $200 \mathrm{~Hz}$ with a hopping height of $1 \mu \mathrm{m}$. Correlative SICM/SOFI data was recorded at $180 \mathrm{~s}$ per frame. The white arrows indicate the corresponding features in SICM and SOFI images. c 3D render of SICM height map aligned to the full SOFI image at the start of the recording $(0 \mathrm{~min})$ revealing the correlative dynamics of actinin in a livecell (Supplementary Movie 2). This time-lapse sequence is representative of more than 5 independent correlative SICM/SOFI time-lapse experiments.

reflecting from a sample at the critical angle. Axial positioning is achieved with a nano-positioning stage (Nano-Drive, MadCityLabs) using a custom-written software in LabVIEW environment. Lateral sample position is controlled by a Scanplus IM $120 \times 80$ (Marzheuser) stage. Image sequence acquisition is done in Micromanager software.

3D widefield imaging setup. Multiplane SOFI imaging was performed on a home-built widefield microscope (Supplementary Fig. 15) with a simultaneous 8plane detection system using an image-splitting prism ${ }^{51}$. Four laser lines were used for illumination: a $120 \mathrm{~mW} 405 \mathrm{~nm}$ laser (iBeam smart, Toptica), an $800 \mathrm{~mW}$ $635 \mathrm{~nm}$ laser (MLL-III-635, Roithner Lasertechnik), $200 \mathrm{~mW} 488 \mathrm{~nm}$ laser (iBEAM-SMART-488-S-HP, Toptica Photonics) and an $800 \mathrm{~mW} 532 \mathrm{~nm}$ laser (MLL-FN-532, Roithner Lasertechnik). The beam of the $635 \mathrm{~nm}$ laser is flat fielded by coupling it into the multimode fiber. All laser lines are collimated, expanded and focused in the back focal plane of the water immersion 60x objective (Olympus UPLSAPO 60XW 1.2 NA). The fluorescence signal is then filtered using a dichroic mirror (zt405/488/532/640/730 rpc, Chroma) and quad band emission filter (405/ 488/532/640 m Chroma). Additional band-pass emission filters (582/75 or 685/70, Chroma) were used for sequential two-color imaging. An image-splitting prism was placed behind the last lens and splits the signal into 8 images recorded by two synchronized sCMOS cameras (ORCA Flash 4.0, Hamamatsu; back projected pixel size of $111 \mathrm{~nm}$ ). Each image plane is equally spaced $350 \mathrm{~nm}$ apart, resulting in a total imaging volume of $50 \times 60 \times 2.45 \mu^{3}$. The sample is positioned in XYZ by using a hybrid piezo nanopositioning stage (3-PT-60-F2,5/5) and MotionCommander-Piezo controller (Nanos Instruments $\mathrm{GmbH}$ ). Synchronization of cameras, imaging acquisition and general setup control is done with a customwritten software in LabVIEW environment.

Scanning ion-conductance microscopy setup. Scanning probe microscopy was performed with a custom-made scanning ion conductance microscope. The sample was actuated in $\mathrm{X}$ and $\mathrm{Y}$ by a piezo-stage (piezosystem Jena TRITOR102SG). The nanocapillary was moved in $\mathrm{Z}$ by a home-built actuator ${ }^{32}$, operated in hopping mode. Borosilicate and quartz nanopipettes were fabricated with a CO-2 laser puller (Model P-2000, Sutter Instruments) with a radius below $60 \mathrm{~nm}^{52}$ (Supplementary Fig. 16).

\section{Combined 2D widefield fluorescence /SICM setup. A home-built widefield} setup is assembled in combination with a SICM scanner (For more detail see SICM setup) mounted atop an inverted Olympus IX-71 microscope body (Supplementary Fig. 17). For sample excitation, a four-color $(405 \mathrm{~nm}, 488 \mathrm{~nm} 561 \mathrm{~nm}, 647 \mathrm{~nm})$ pigtailed Monolithic Laser Combiner (400B, Agilent Technologies) is used. Light is collimated and focused to the back focal plane of the oil-immersion high-NA objective (Olympus TIRFM 100x, 1.45 NA) by using a custom built TIRF illuminator. The fluorescence signal is then filtered using a dichroic mirror $(493 / 574 \mathrm{~nm}$ BrightLine, Semrock) and a band emission filter (405/488/568 nm stop line, Semrock). Finally, the light is focused on an sCMOS camera (Photometrics, Prime $95 \mathrm{~B}$; back projected pixel size of $111 \mathrm{~nm}$ ). Coarse lateral sample positioning is done with a mechanical stage, while fine positioning is achieved with a SICM XY piezo scanner (Piezosystem Jena TRITOR102SG). Image stacks for SOFI are recorded with a Micromanager software, while laser control and sample positioning are achieved in custom-written LabVIEW software.
Coverslip fabrication. High precision No. 1.5 borosilicate $25 \mathrm{~mm}$ coverslips (Marienfeld) were patterned with a custom layout (Supplementary Fig. 18) by using a commercial UV excimer laser patterning setup (PTEC LSV3). It allowed to create a user-friendly sample map, which was crucial for further SOFI-SICM correlation experiments while transferring the sample between different setups. After patterning, coverslips were cleaned with piranha solution, washed in MiliQ water, dried with $\mathrm{N}_{2}$ flow and kept dry for further use. Before use, coverslips were cleaned with oxygen plasma cleaner (Femto A, Diener electronic $\mathrm{GmbH}$ ) for $660 \mathrm{~s}$ at maximum power setting, washed with $\mathrm{PBS}(\mathrm{pH}=7.4)$ once and coated with 50 $\mu \mathrm{M}$ fibronectin solution in $\mathrm{PBS}(\mathrm{pH}=7.4)$ for $30 \mathrm{~min}$ at $37^{\circ} \mathrm{C}$ before seeding the cells.

Cell culture. COS-7 cells (ECACC 87021302) obtained from ECACC were used. Cells were cultured at $37{ }^{\circ} \mathrm{C}$ and $5 \% \mathrm{CO}_{2}$. DMEM high glucose without phenol red medium (Gibco, Thermo Fisher Scientific) was used, containing 10\% fetal bovine serum (Gibco, Thermo Fisher Scientific), 1\% penicillin-streptomycin (Gibco, Thermo Fisher Scientific) and $4 \mathrm{mM}$ L-glutamine (Gibco, Thermo Fisher Scientific). Before seeding on coverslips, cells were detached from a flask with TrypLE (Gibco, Thermo Fisher Scientific) and 30000 cells were seeded on $25 \mathrm{~mm}$ coverslips coated with fibronectin. Coverslips were washed twice with PBS before seeding the cells and $2 \mathrm{ml}$ of DMEM medium was used. Cells were grown overnight $(12-16 \mathrm{~h})$ before fixation in 6-well plates.

Sample fixation and staining. Cells were washed once with DMEM described previously and incubated for $90 \mathrm{~s}$ in a prewarmed microtubule extraction buffer, consisting of $80 \mathrm{mM}$ PIPES, $7 \mathrm{mM} \mathrm{MgCl} 2,1 \mathrm{mM}$ EDTA, $150 \mathrm{mM} \mathrm{NaCl}$ and $5 \mathrm{mM}$ D-glucose with a pH adjusted to 6.8 using $\mathrm{KOH}$ with $0.3 \%$ (v/v) Triton X-100 (AppliChem) and $0.25 \%(\mathrm{v} / \mathrm{v})$ EM-grade glutaraldehyde (Electron Microscopy Sciences). After $90 \mathrm{~s}$ the solution was exchanged to pre-warmed $4 \%$ paraformaldehyde dissolved in PBS $(\mathrm{pH}=7.4)$ and samples were incubated for $10 \mathrm{~min}$ at room temperature. Afterwards, samples were washed thrice for $5 \mathrm{~min}$ with PBS on orbital shaker. Cells were kept for 5 min with a freshly prepared $10 \mathrm{mM} \mathrm{NaBH}_{4}$ solution in PBS on an orbital shaker in order to reduce background fluorescence. Step was followed by one quick wash in PBS, and two washes of 10 min in PBS on an orbital shaker. Samples were then additionally permeabilized to ensure antibody penetration with $0.1 \%(\mathrm{v} / \mathrm{v})$ Triton X-100 in PBS $(\mathrm{pH}=7.4)$ on an orbital shaker followed by additional wash with PBS. Finally, samples were blocked with freshly prepared blocking buffer consisting of $2 \%$ (w/v) BSA, $10 \mathrm{mM}$ glycine, $50 \mathrm{mM}$ ammonium chloride $\mathrm{NH}_{4} \mathrm{Cl}$ in $\mathrm{PBS}(\mathrm{pH}=7.4)$ for $60 \mathrm{~min}$ at room temperature or stored overnight at $4{ }^{\circ} \mathrm{C}$ for further staining. All chemicals were bought from Sigma Aldrich unless stated differently.

Two-color sample staining. For incubation with antibodies and/or phalloidin, coverslips were placed on parafilm in a closed box in the dark, at high humidity to prevent coverslips from drying. $100 \mu \mathrm{L}$ of staining solution was typically used for each coverslip. After blocking, samples were incubated with primary anti-tubulin antibody (clone B-5-1-2, Sigma-Aldrich, 1:50 dilution) in blocking buffer for 60 $\mathrm{min}$ at room temperature. Samples were washed with blocking buffer thrice for 5 min on orbital shaker. Coverslips were incubated with secondary donkey antimouse-Abberior FLIP-565 antibody (1:50 dilution), which was labeled as described previously ${ }^{14}$. Samples then were kept in blocking buffer for $60 \mathrm{~min}$ and washed thrice for $5 \mathrm{~min}$ on orbital shaker. Samples were incubated for $10 \mathrm{~min}$ in $2 \%(\mathrm{w} / \mathrm{v})$ 
PFA in PBS ( $\mathrm{pH}=7.4$ ) as a post-fixation step followed by three 5 min washes with PBS on orbital shaker. After tubulin staining, actin was stained with $500 \mathrm{nM}$ custom synthesized phalloidin-f-HM-SiR (Supplementary Fig. 19) or phalloidinAlexa-647 (Sigma Aldrich) solutions in PBS by incubating for $60 \mathrm{~min}$ at room temperature. Samples were washed thrice for 5 min with PBS on orbital shaker and imaged immediately in imaging buffer.

Imaging buffers. 2D imaging with self-blinking dyes was performed in $50 \%$ glycerol PBS solution at $\mathrm{pH}=8$. The buffer was degassed with $\mathrm{N}_{2}$ flow for $30 \mathrm{~min}$ before use. 3D phalloidin-actin imaging was performed in an imaging buffer described previously ${ }^{53}$ containing $10 \%$ (w/v) D-glucose, $20 \%$ (v/v) glycerol, $50 \mathrm{mM}$ TRIS, $10 \mathrm{mM} \mathrm{NaCl}, 2 \mathrm{mM}$ COT, $10 \mathrm{mM}$ MEA, $2.5 \mathrm{mM}$ PCA and $50 \mathrm{nM}$ PCD with a $\mathrm{pH}$ adjusted with $\mathrm{HCl}$ to 7.5 . 3D imaging of tubulin labeled with an Abberior FLIP-565 was performed in 50\% (v/v) glycerol solution in PBS $(\mathrm{pH}=7.4)$. All imaging experiments were done in a sealed imaging chamber.

Two-color SOFI imaging. 2D SOFI imaging was performed with self-blinking dyes sequentially. Phalloidin-f-HM-SiR labeled actin was imaged first at $275 \mathrm{~W} / \mathrm{cm}^{2}$ $635 \mathrm{~nm}$ excitation, then $680 \mathrm{~W} / \mathrm{cm}^{2}$ of $561 \mathrm{~nm}$ was used to image Abberior FLIP565 labeled tubulin. The sample was kept on the setup for at least $30 \mathrm{~min}$ before imaging, to reduce thermomechanical drift during imaging. 16,000-30,000 frames were acquired for each channel for high-order SOFI analysis with a minimal photobleaching effects (Supplementary Figs. 3 and 20). Sample drift between twocolor acquisitions was neglected for further analysis. 3D SOFI imaging was first performed with phalloidin-Alexa-647 labeled actin excited with a $632 \mathrm{~nm}$ laser at $3.4 \mathrm{~kW} / \mathrm{cm}^{2}$ with a low power $\left(5 \mathrm{~W} / \mathrm{cm}^{2}\right) 405 \mathrm{~nm}$ activation laser to increase the population of fluorophores in a bright state. After imaging multiple cells, the oxygen-scavenging imaging buffer was changed to $50 \%$ (v/v) glycerol in PBS for Abberior FLIP-565 labeled tubulin imaging. The sample was then imaged with the $532 \mathrm{~nm}$ laser at $3.5 \mathrm{~kW} / \mathrm{cm}^{2} .4000$ frames for each image plane were recorded for high-order 3D-SOFI analysis. After recording each image stack, a subsequent brightfield-image was taken for a further alignment of 2-channel data. $50 \mathrm{~ms}$ exposure time was used for all imaging experiments. The data presented in the paper are from two distinct samples.

SICM imaging and processing. The SICM imaging was performed in PBS $(\mathrm{pH}=$ 7.4). On fixed cells, SICM images with $1024 \times 1024$ pixels were acquired at a pixel acquisition rate of $200 \mathrm{~Hz}$ with a hopping height of 5 and $6 \mu \mathrm{m}$. After performing fluorescence imaging corresponding cells were found using markers described in a coverslip fabrication section. On live cells, SICM images with $512 \times 256$ and $200 \times$ 100 pixels were acquired at a pixel acquisition rate of $200 \mathrm{~Hz}$ with a hopping height of 1 and $3 \mu \mathrm{m}$. These experiments were performed in the combined SICM/SOFI system described previously. The current setpoint used in all the SICM measurements was $99 \%$ of the normalized current recorded. SICM images were further processed using Gwyddion ${ }^{54}$ and Fiji ${ }^{55}$.

SOFI Image analysis and image alignment. 2D and 3D SOFI image analysis was performed as described previously 14,51 . Briefly, the image stack was drift-corrected using cross-correlation between SOFI sub-sequences before performing cumulant calculation. Different planes of the 3D image stack were registered based on acquired bead stack and images sequence was drift-corrected after the registration, assuming that the drift is homogenous within the volume. $4^{\text {th }}$ order SOFI images were used in 2D SOFI case, while $3^{\text {rd }}$ order SOFI images were taken for 3D SOFI. Images were further deconvolved with a Lucy-Richardson deconvolution algorithm using gaussian PSF model. Deconvolution settings were optimized using decorrelation analysis algorithm for resolution estimation ${ }^{30} .2 \mathrm{D}$ and $3 \mathrm{D}$ SOFI analysis code was implemented in Matlab and is available upon request. After 2D and 3D SOFI processing, images were aligned with a topographical SICM map using custom-written Python code based on the hand-selected features in the actin channel and SICM image using an affine transformation (Supplementary Fig. 6). For two-color 3D SOFI, two-color SOFI stacks were co-registered based on brightfield images and then aligned with SICM image based on the actin channel.

Live-cell SOFI/SICM imaging. COS-7 cells were seeded according to the procedure described previously. After $12-16 \mathrm{~h}$ cells were transfected with actinin-mEOS2 or Lifeact-mEOS2 (Supplementary Table 1) with a Lipofectamine 3000 (Thermo Fisher) according to the protocol provided by manufacturer. Cells were used for combined SICM-SOFI experiment $24 \mathrm{~h}$ after the transfection. Imaging was performed at $37^{\circ} \mathrm{C}$ and $5 \% \mathrm{CO}_{2}$ in a custom-built imaging chamber in FluoroBrite DMEM media (Gibco, Thermo Fisher Scientific) in order to reduce autofluorescence. SICM and SOFI imaging was performed subsequently i.e., after each SICM image, a fluorescent stack of 300 frames with an exposure time of $50 \mathrm{~ms}$ was recorded. The first 50 frames were excluded from SOFI processing due to rapid intensity change upon 405 activation and only 250 frames were used for further SOFI analysis. Live-cell imaging was performed under the low-illumination intensity $\left(500 \mathrm{~W} / \mathrm{cm}^{2}\right.$ of 561 imaging laser and $0.3 \mathrm{~W} / \mathrm{cm}^{2}$ of $405 \mathrm{~nm}$ activation laser) in order to reduce phototoxicity.
Correlative 3D SICM/SOFI data rendering. In order to fully expose the correlative 3D data, we have used the advanced open-source 3D rendering tool Blender 3D for data visualization. Normalized topographical SICM data was imported as a height map and scaled in the axial direction. Then, 2D SOFI data was overlaid by importing a co-registered fluorescence image and a custom-written shader was used for volumetric multiplane data rendering (Supplementary Fig. 21) to generate the final figures.

Reporting summary. Further information on research design is available in the Nature Research Reporting Summary linked to this article.

\section{Data availability}

Source Data are provided as a Source Data file. RAW and processed image data generated in this study are available online: https://srm.epfl.ch/DatasetPage?name=SICM_SOFI_ dataset. Source data are provided with this paper.

\section{Code availability}

The 2D/3D SOFI code used in this study are available: https://github.com/kgrussmayer/ sofipackage

Received: 23 October 2020; Accepted: 14 July 2021; Published online: 27 July 2021

\section{References}

1. Pollard, T. D. Cell motility and cytokinesis: From mysteries to molecular mechanisms in five decades. Annu. Rev. Cell Dev. Biol. 35, 1-28 (2019)

2. Sánchez Alvarado, A. \& Yamanaka, S. Rethinking differentiation: stem cells, regeneration, and plasticity. Cell 157, 110-119 (2014).

3. Derby, M. C. \& Gleeson, P. A. New insights into membrane trafficking and protein sorting. Int. Rev. Cytol. 261, 47-116 (2007).

4. Alberts, B. et al. General Principles of Cell Communication. Garland Science (2002).

5. Ban, N., Nissen, P., Hansen, J., Moore, P. B. \& Steitz, T. A. The complete atomic structure of the large ribosomal subunit at $2.4 \AA$ resolution. Science. 289, 905-920 (2000).

6. Fang, Q. et al. Near-atomic structure of a giant virus. Nat. Commun. 10, 1-11 (2019).

7. Bharat, T. A. M. \& Kukulski, W. Cryo-correlative light and electron microscopy. Wiley (2019).

8. Neumüller, J. Electron tomography-a tool for ultrastructural 3D visualization in cell biology and histology. Wien. Medizinische Wochenschr. 168, 322-329 (2018).

9. Sahl, S. J., Hell, S. W. \& Jakobs, S. Fluorescence nanoscopy in cell biology. Nat. Rev. Mol. Cell Biol. 18, 685-701 (2017).

10. Waldchen, S., Lehmann, J., Klein, T., Van De Linde, S. \& Sauer, M. Lightinduced cell damage in live-cell super-resolution microscopy. Sci. Rep. 5, 1-12 (2015).

11. Dertinger, T., Colyer, R., Iyer, G., Weiss, S. \& Enderlein, J. Fast, backgroundfree, 3D super-resolution optical fluctuation imaging (SOFI). Proc. Natl Acad. Sci. U. S. A. 106, 22287-22292 (2009).

12. Geissbuehler, S., Dellagiacoma, C. \& Lasser, T. Comparison between SOFI and STORM. Biomed. Opt. Express 2, 408 (2011).

13. Geissbuehler, S. et al. Live-cell multiplane three-dimensional super-resolution optical fluctuation imaging. Nat. Commun. 5, 1-7 (2014).

14. Grußmayer, K., Lukes, T., Lasser, T. \& Radenovic, A. Self-blinking dyes unlock high-order and multiplane super-resolution optical fluctuation imaging. ACS Nano 14, 9156-9165 (2020).

15. Shibata, M., Watanabe, H., Uchihashi, T., Ando, T. \& Yasuda, R. High-speed atomic force microscopy imaging of live mammalian cells. Biophys. Physicobiol. 14, 127-135 (2017).

16. Moreno Flores, S. \& Toca-Herrera, J. L. The new future of scanning probe microscopy: Combining atomic force microscopy with other surface-sensitive techniques, optical microscopy and fluorescence techniques. Nanoscale $\mathbf{1}$, 40-49 (2009).

17. Krieg, M. et al. Atomic force microscopy-based mechanobiology. Nat. Rev. Phys. 1, 41-57 (2019).

18. Frederix, P. L. T. M., Bosshart, P. D. \& Engel, A. Atomic force microscopy of biological membranes. Biophysical J. 96, 329-338 (2009).

19. Nievergelt, A. P., Banterle, N., Andany, S. H., Gönczy, P. \& Fantner, G. E. Highspeed photothermal off-resonance atomic force microscopy reveals assembly routes of centriolar scaffold protein SAS-6. Nat. Nanotechnol. 13, 696-701 (2018).

20. Odermatt, P. D. et al. High-resolution correlative microscopy: bridging the gap between single molecule localization microscopy and atomic force microscopy. Nano Lett. 15, 4896-4904 (2015). 
21. Gómez-Varela, A. I. et al. Simultaneous co-localized super-resolution fluorescence microscopy and atomic force microscopy: combined SIM and AFM platform for the life sciences. Sci. Rep. 10, 1-10 (2020).

22. Seifert, J., Rheinlaender, J., Novak, P., Korchev, Y. E. \& Schäffer, T. E. Comparison of atomic force microscopy and scanning ion conductance microscopy for live cell imaging. Langmuir 31, 6807-6813 (2015).

23. Hansma, P. K., Drake, B., Marti, O., Gould, S. A. \& Prater, C. B. The scanning ion-conductance microscope. Science 243, 641-643 (1989).

24. Novak, P. et al. Nanoscale live-cell imaging using hopping probe ion conductance microscopy. Nat. Methods 6, 279-281 (2009).

25. Rheinlaender, J. \& Schäffer, T. E. Image formation, resolution, and height measurement in scanning ion conductance microscopy. J. Appl. Phys. 105, 094905 (2009).

26. Hagemann, P., Gesper, A. \& Happel, P. Correlative stimulated emission depletion and scanning ion conductance microscopy. ACS Nano 12, 5807-5815 (2018).

27. Steinbock, L. J., Steinbock, J. F. \& Radenovic, A. Controllable shrinking and shaping of glass nanocapillaries under electron irradiation. Nano Lett. 13, 1717-1723 (2013)

28. Deschout, H. et al. Complementarity of PALM and SOFI for super-resolution live-cell imaging of focal adhesions. Nat. Commun. 7, 13693 (2016).

29. Werther, P. et al. Live-cell localization microscopy with a fluorogenic and selfblinking tetrazine probe. Angew. Chem. Int. Ed. 59, 804-810 (2020).

30. Descloux, A., Grußmayer, K. S. \& Radenovic, A. Parameter-free image resolution estimation based on decorrelation analysis. Nat. Methods 16 918-924 (2019).

31. Ovesný, M., Křížek, P., Borkovec, J., Svindrych, Z. \& Hagen, G. M. ThunderSTORM: a comprehensive ImageJ plug-in for PALM and STORM data analysis and super-resolution imaging. Bioinformatics 30, 2389-2390 (2014).

32. Leitao, S. M. et al. Time-resolved scanning ion conductance microscopy for three-dimensional tracking of nanoscale cell surface dynamics. Preprint at https://www.biorxiv.org/content/10.1101/2021.05.13.444009v1 (2021).

33. Henderson, E. \& Sakaguchi, D. S. Imaging F-actin in fixed glial cells with a combined optical fluorescence/atomic force microscope. Neuroimage 1, 145-150 (1993).

34. Geissbuehler, S. et al. Live-cell multiplane three-dimensional super-resolution optical fluctuation imaging. Nat. Commun. 5, 5830 (2014).

35. Berdyyeva, T., Woodworth, C. D. \& Sokolov, I. Visualization of cytoskeletal elements by the atomic force microscope. Ultramicroscopy 102, 189-198 (2005).

36. Odermatt, P. D. et al. High-resolution correlative microscopy: bridging the gap between single molecule localization microscopy and atomic force microscopy. Nano Lett. 15, 4896-4904 (2015).

37. Cosentino, M., Canale, C., Bianchini, P. \& Diaspro, A. AFM-STED correlative nanoscopy reveals a dark side in fluorescence microscopy imaging. Sci. Adv. 5, eaav8062 (2019).

38. Tanaka, A. et al. Time-lapse imaging of morphological changes in a single neuron during the early stages of apoptosis using scanning ion conductance microscopy. J. Struct. Biol. 191, 32-38 (2015)

39. Gorelik, J. et al. Scanning surface confocal microscopy for simultaneous topographical and fluorescence imaging: application to single virus-like particle entry into a cell. Proc. Natl Acad. Sci. USA. 99, 16018-16023 (2002).

40. Shevchuk, A. I. et al. Imaging single virus particles on the surface of cell membranes by high-resolution scanning surface confocal microscopy. Biophys. J. 94, 4089-4094 (2008)

41. Rheinlaender, J. \& Schäffer, T. E. Lateral resolution and image formation in scanning ion conductance microscopy. Anal. Chem. 87, 7117-7124 (2015).

42. Garcia, R. Nanomechanical mapping of soft materials with the atomic force microscope: methods, theory and applications. Chem. Soc. Rev. 49, 5850-5884 (2020).

43. Hinterdorfer, P. \& Dufrêne, Y. F. Detection and localization of single molecular recognition events using atomic force microscopy. Nat. Methods 3 , 347-355 (2006).

44. Yamada, K. M. \& Sixt, M. Mechanisms of 3D cell migration. Nat. Rev. Mol. Cell Biol. 20, 738-752 (2019).

45. Klausen, L. H., Fuhs, T. \& Dong, M. Mapping surface charge density of lipid bilayers by quantitative surface conductivity microscopy. Nat. Commun. 7, 12447 (2016)

46. Johannes, L., Pezeshkian, W., Ipsen, J. H. \& Shillcock, J. C. Clustering on membranes: fluctuations and more. Trends Cell Biol. 28, 405-415 (2018).

47. Yesylevskyy, S. O., Rivel, T. \& Ramseyer, C. The influence of curvature on the properties of the plasma membrane. Insights from atomistic molecular dynamics simulations. Sci. Rep. 7, 1-13 (2017).

48. Sezgin, E., Levental, I., Mayor, S. \& Eggeling, C. The mystery of membrane organization: composition, regulation and roles of lipid rafts. Nat. Rev. Mol Cell Biol. 18, 361-374 (2017).
49. Heine, M., Ciuraszkiewicz, A., Voigt, A., Heck, J. \& Bikbaev, A. Surface dynamics of voltage-gated ion channels. Channels 10, 267-281 (2016).

50. Deschamps, J., Rowald, A. \& Ries, J. Efficient homogeneous illumination and optical sectioning for quantitative single-molecule localization microscopy. Opt. Express 24, 28080 (2016).

51. Descloux, A. et al. Combined multi-plane phase retrieval and super-resolution optical fluctuation imaging for 4D cell microscopy. Nat. Photonics 12, 165-172 (2018).

52. Navikas, V. et al. High-throughput, nanocapillary filling method based on microwave radiation. ACS Appl. Nano Mater. 3, 7829-7834 (2020).

53. Olivier, N., Keller, D., Gönczy, P. \& Manley, S. Resolution doubling in 3D-STORM imaging through improved buffers. PLoS One 8, e69004 (2013).

54. Nečas, D. \& Klapetek, P. Gwyddion: an open-source software for SPM data analysis. Open Phys. 10, 181-188 (2012)

55. Schindelin, J. et al. Fiji: an open-source platform for biological-image analysis. Nat. Methods 9, 676-682 (2012).

\section{Acknowledgements}

V.N. and A.R. acknowledges the support of the Max Planck-EPFL Center for Molecular Nanoscience and Technology and Swiss National Science Foundation through the National Centre of Competence in Research Bio-Inspired Materials. K.Y. and D.P.H gratefully acknowledge funding by the Centre of Membrane Proteins and Receptors (COMPARE, Universities of Birmingham and Nottingham). G.E.F. acknowledges the support by the European Research Council under grant number ERC-2017-CoG; InCell; Project number 773091. The authors thank Adrian Pascal Nievergelt and Charlène Brillard for their input on technical development of SICM controller. The authors also thank Prof. Jens Anders and his team from the University of Stuttgart for insightful discussions into high-speed transimpedance amplifiers.

\section{Author contributions}

V.N. and S.L. equally contributed to the paper by conceiving and refining general idea and writing the final manuscript with input from all the authors. V.N. prepared the cell samples, performed all optical imaging experiments and correlative SOFI image data analysis and the final data visualization. S.L. performed the SICM imaging and SICM data processing. B.D and S.L. built the SICM setup. Simultaneous SICM/SOFI imaging was performed by V.N. and S.L. K.G. contributed by creating and adapting labeling protocols. A.D. adapted and improved the existing SOFI analysis code. K.Y. and P.W. synthesized and provided f-HM-SiR-phalloidin conjugate. D.-P.H., R.W., A.R and G.E.F. supervised the work.

\section{Competing interests}

The authors declare no competing interests.

\section{Additional information}

Supplementary information The online version contains supplementary material available at https://doi.org/10.1038/s41467-021-24901-3.

Correspondence and requests for materials should be addressed to A.R. or G.E.F.

Peer review information Nature Communications thanks Jörg Enderlein, Patrick Happel and Yuri Korchev for their contribution to the peer review of this work.

Reprints and permission information is available at http://www.nature.com/reprints

Publisher's note Springer Nature remains neutral with regard to jurisdictional claims in published maps and institutional affiliations.

\section{(i)}

Open Access This article is licensed under a Creative Commons Attribution 4.0 International License, which permits use, sharing, adaptation, distribution and reproduction in any medium or format, as long as you give appropriate credit to the original author(s) and the source, provide a link to the Creative Commons license, and indicate if changes were made. The images or other third party material in this article are included in the article's Creative Commons license, unles indicated otherwise in a credit line to the material. If material is not included in the article's Creative Commons license and your intended use is not permitted by statutory regulation or exceeds the permitted use, you will need to obtain permission directly from the copyright holder. To view a copy of this license, visit http://creativecommons.org/ licenses/by/4.0/

(C) The Author(s) 2021 